\title{
Orientaciones preliminares para la preservación digital del patrimonio documental arquitectónico de Tampico
}

\author{
David Alonso Leija Román* \\ Reina Loredo Cansino* \\ Lorena Gertrudis Valle Chavarria*
}

Artículo recibido:

27 de septiembre de 2019

Artículo aceptado:

11 de marzo de 2020

Artículo de investigación

\section{Resumen}

En este artículo se abordan las consideraciones para preparar un punto de partida preliminar en la planificación de actividades de preservación digital de una colección documental física, referente al patrimonio arquitectónico moderno alojada en el Archivo Histórico de Tampico (AHT) "Carlos González Salas", de Tamaulipas, México. Se estudia desde la perspectiva de caso si la demanda de una comunidad designada y la observación de los aspectos nucleares que intervienen en las actividades de preservación digital -infraestructura organizacional, infraestructura tecnológica y recursos requeridos- contribuyen útilmente con información orientativa preliminar para la planificación de políticas, estrategias y acciones de preservación digital

* Facultad de Arquitectura, Diseño y Urbanismo, Universidad Autónoma de Tamaulipas, México dleija@docentes.uat.edu.mx rloredoc@docentes.uat.edu.mx Ivalle@docentes.uat.edu.mx

INVESTIGACIÓN BIBLIOTECOLÓGICA, vol. 34, núm. 85, octubre/diciembre, 2020, México, ISSN: 2448-8321 pp. 13-32 
de colecciones documentales de interés en una comunidad específica. Se discuten consideraciones mínimas y prioritarias de preservación digital de acuerdo con la disposición de colecciones, recursos humanos, infraestructura tecnológica y organización institucional disponible en el AHT para situar qué actividades preliminares de tipo ejecutivo, operativo y técnico están a su alcance de acuerdo con las recomendaciones de buenas prácticas a nivel nacional e internacional en materia de preservación digital a largo plazo.

Palabras clave: Patrimonio Documental; Preservación Digital; Patrimonio Arquitectónico Documental

\title{
Preliminary guidelines for digital preservation of Tampico's architectural documentary heritage David Alonso Leija Román, Reina Loredo Cansino and Lorena Gertrudis Valle Chavarria
}

\begin{abstract}
This article addresses the considerations to prepare a starting point in the planning of digital preservation activities for a physical documentary collection regarding the modern architectural heritage housed in the Historical Archive of Tampico (AHT) "Carlos González Salas" of Tamaulipas, México. The question is, from the case perspective, whether the demand of a designated community and the observation of the nuclear aspects involved in digital preservation activities -organizational infrastructure, technological infrastructure and required resources- contribute usefully as preliminary guidance in the planning of policies, strategies and actions of digital preservation of documentary collections of interest in a specific community. Minimum and priority considerations of digital preservation are discussed in accordance with the provision of collections, human resources, technological infrastructure and institutional organization available in the AHT, in order to place which preliminary executive, operational and technical activities are within your reach in accordance with the recommendations of good practices at national and international level regarding long-term digital preservation.
\end{abstract}

Keywords: Documentary Heritage; Digital Preservation; Documentary Architectural Heritage 


\section{INTRODUCCIÓN}

\section{Objetivo}

— l objetivo de este documento es comprobar si la propuesta metodológica Edel modelo de orientación preliminar e bitos para la gestión de preservación digital, que considera el estudio de actividades de identificación, interés y demanda de una comunidad designada ante una colección documental específica -sobre arquitectura moderna de Tampico-, y la observación de aspectos nucleares de la preservación digital -infraestructura organizacional, tecnológica y recursos disponibles- contribuyen en la generación de información orientativa para perfilar de manera preliminar la planificación de políticas, procedimientos y la definición de tecnología para un programa de preservación digital de colecciones documentales pertenecientes a un archivo municipal con recursos limitados y aspiración de transformación digital de su patrimonio documental físico, de acuerdo con las buenas prácticas y posibilidades a nivel ejecutivo, operativo y técnico. Este trabajo ofrece una línea general de orientación para la unión de recursos disponibles y consideración de necesidades de un archivo, para encontrar una compatibilidad de soluciones alcanzables y de primera mano a sus condiciones e infraestructura. De manera indirecta, se evidencia el alcance de operación institucional por el que atraviesa un archivo del interior -provincia- de México con responsabilidades intrínsecas, extrínsecas y obligadas de salvaguardar el patrimonio documental de su comunidad, exponiendo sus límites como organización, en formación y redes de cooperación para afrontar los retos actuales y futuros para su labor de gestión documental digital como institución. Los factores institucionales -viabilidad institucional, sostenibilidad económica y falta de preparación en la organización- y no los tecnológicos son considerados los principales obstáculos para las actividades encaminadas a la preservación digital (Keefer Riva y Gallart Marsillas, 2007).

\section{La preservación digital del patrimonio documental en bibliotecas, archivos y museos (BAM) de México}

Los documentos y objetos digitales gestionados por archivos, bibliotecas, editoriales e instituciones con patrimonio cultural e histórico crecen exponencialmente día a día, y encuentran retos heterogéneos -técnicos, organización, legales, de sostenibilidad, etc.- para integrar ecosistemas de confianza para la consulta y acceso a sus fondos digitales (Voutssas, 2010). La preservación digital es una actividad que integra políticas, estrategias y acciones 
técnicas para mantener los contenidos digitales de forma accesible a lo largo del tiempo (Leija, 2017). Los objetos y contenidos digitales pueden ser de extremadamente breve duración de vida a menos que se les preste la atención adecuada para preservarlos con integridad a largo plazo. En la gestión documental digital, los conceptos de autenticidad y confianza son fundamentales para la integridad digital, ya que éstos definen si desde su origen los documentos digitales -nacidos digitales o digitalizados- serán confiables tanto para su acceso como para su preservación a largo plazo (Corrado, 2019). En la práctica, instituciones de memoria y patrimonio histórico trabajan con modelos de preservación digital centralizados, distribuidos y colaborativos para planificar flujos de trabajo humano y actividades técnicas que les permitan crear, recibir, transformar y mantener colecciones digitales a largo plazo. El auge de la digitalización de documentos en instituciones BAM -bibliotecas, archivos y museos- se adoptó como parte de la transformación y solución para crear copias digitales de objetos físicos en las pasadas décadas. No obstante, la implementación de procesos de digitalización masiva de documentos históricos y culturales trajo consigo nuevos retos en el ámbito digital, como lo son el almacenamiento seguro, el acceso continuo, la corrupción espontánea, la migración, etc. Algunos de estos retos han hecho que los criterios de digitalización de documentos normalizados -ISO 19264, FADGI y Metamorfoze- sean redefinidos porque una gran cantidad de objetos digitalizados han sufrido pérdidas de calidad e información, ya que su prioridad de creación se enfocaba en la difusión y el acceso público, y no a procurar estándares de calidad para su preservación a largo plazo. La digitalización puso sobre la mesa la necesidad de distinguir funcionalmente entre la conservación digital, la seguridad informática y la preservación digital; cuando hay una correcta combinación y distinción de éstas es posible asegurar una gestión integral y un acceso seguro a los contenidos digitales a largo plazo (Térmens Graells, 2013).

Recomendaciones y normas especializadas en buenas prácticas de preservación digital como ISO, el modelo de referencia OAIS 14721:2012 (Open Archival Information System, https://www.iso.org/standard/57284.html) y la matriz de recomendaciones de la National Digital Stewaredship Alliance (NDSA Levels, http://ndsa.org/activities/levels-of-digital-preservation) sugieren orientar las prácticas de proyectos y programas de preservación digital con líneas de alcance a la aplicación de políticas, estrategias y acciones técnicas para su implementación paulatina en modo de autoevaluación de objetivos. De acuerdo con Hirtle (2008), la preservación digital tiene más de 20 años de vida (desde 1994) como concepto aceptado y como actividad formal aplicada a la gestión de información y documentación digitalizada y nacida 
digitalmente en bibliotecas de Norteamérica. En este contexto, es necesario situar que la preservación digital es la finalidad y aspiración de toda gestión, curaduría o difusión digital de objetos digitales de archivos patrimoniales. El colectivo de instituciones de memoria y organizaciones agrupadas en el acrónimo GLAMUR -Galerie, Library, Archive, Museum, University, Repository-, traducido como BAMG -bibliotecas, archivos, museos y galerías-, se coloca como pionero en iniciativas con procesos de digitalización, depósito y preservación digital de archivos documentales. Los métodos y técnicas de preservación digital empleadas por este tipo de instituciones mantienen una afinidad de enfoque tecnológico, pues la finalidad es procurar la integridad y autenticidad de objetos digitales (Rodríguez, 2019). En este sentido, los esfuerzos y experiencias en instituciones de memoria siguen siendo contrastantes y parciales, ya que las dificultades a nivel técnico, operativo, presupuestal y de colaboración inciden directamente en su organización, operación y mantenimiento con fines de preservación de la información digital (Arreola Semadeni, 2012).

En instituciones de Latinoamérica y en gran parte del panorama mundial los alcances en acciones de preservación digital son variables en dimensiones ejecutivas, operativas y técnicas, ya que no todas las instituciones, fuera de los ámbitos centralizados de capitales del mundo, cuentan con los recursos establecidos en las buenas prácticas internacionales específicas que sugieren el desarrollo de políticas organizacionales, sistemas e infraestructura tecnológica de calidad y formación de recursos humanos, que ayuden a afrontar y sostener un reto digital de esta envergadura (Leija y Márdero, 2019). Es de vital importancia observar casos de estudio de archivos municipales y locales para comprender, analizar y definir esquemas de preparación preliminar que ayuden a afrontar el tema y poner en perspectiva sus necesidades con requisitos mínimos para dirigir y orientar un procedimiento de transformación digital de archivo - digitalización, difusión y preservación digital- como eje de aspiraciones en la tarea de todos los archivos digitales del siglo XXI.

\section{El Archivo Histórico de Tampico y su patrimonio documental}

El Archivo Histórico de Tampico (AHT) “Carlos González Salas”, del municipio de Tampico, en el estado mexicano de Tamaulipas, alberga un fondo documental con acervos y colecciones que cuentan la historia de la ciudad desde 1824. El patrimonio documental del AHT está en su mayoría catalogado y organizado en colecciones, de las que destacan la hemeroteca con periódicos de la ciudad de 1885 a 2018, una fonoteca con cerca de 7000 piezas de artistas regionales, actas de cabildo en 120 libros, diarios oficiales, periódicos oficiales 
y cajas del Archivo General de la Nación (AGN) con documentos de la administración pública de Tampico de 1885 a 1975. Dentro de este patrimonio físico y analógico documental, el AHT ha emprendido acciones para digitalizar y almacenar una década de su hemeroteca, del periodo 1941-1950, en dispositivos de almacenamiento externo - discos de estado sólido- sin ninguna política de gestión de acceso, preservación, mantenimiento o estándares de control. En sectores clave y referentes para liderar modelos de gestión de actividades de preservación digital en México, como el sector gubernamental de archivos, se ha advertido en distintas ocasiones sobre la necesidad de establecer un estándar intersectorial o métodos estandarizados para preservar digitalmente diversos conglomerados de información (Voutssás, 2012).

La necesidad de crear y desarrollar un informe de estado de caso, estructuras particulares y definición ejecutiva de políticas de preservación digital del patrimonio histórico de colecciones documentales de arquitectura en Tampico supone revisar y poner en perspectiva las recomendaciones universales sugeridas en las directrices la Unesco de 2003 a través de la carta sobre la preservación del patrimonio digital, para potenciar paulatinamente la agenda de desarrollo digital como referente e impulsor de las necesidades tanto de acceso, vigilancia, acción y continuidad, como de estrategias, políticas, protección, promoción, funciones y alianzas articuladas desde los distintos gobiernos y naciones para la preservación del patrimonio digital como compromiso internacional de participación, tomando en cuenta sus límites.

\section{El patrimonio arquitectónico moderno de Tampico}

Todas las ciudades, sin excepción y dependencia de su alcance geográfico, político o económico, son capaces de generar patrimonio documental de su contexto histórico y cultural, el cual paulatinamente se convierte en archivos de interés para una comunidad. A finales del siglo XIX, con el fin de activar la economía mexicana, el gobierno de Porfirio Díaz promovió la diversificación de la producción nacional, la ampliación del comercio exterior, la explotación de recursos del subsuelo y concedió permisos para la exploración petrolera. En este proyecto de nación, las vías férreas serían los nervios del país y el camino a la integración nacional (Riguzzi, 1999). Las costas del golfo de México fueron los espacios geográficos claves para las actividades intensivas de expansión comercial del interior al exterior de la república mexicana; por su estratégica ubicación, Tampico se convertiría, primero, en uno de los principales puntos de salida para los productos de exportación a partir de los trabajos para la modernización del puerto y la llegada del ferrocarril, y luego, en las primeras décadas del siglo XX, en el mayor centro de explotación, refinación y transporte de petróleo del país (Gamboa Ramírez y Páez Flores, 2012). 
Así, el proceso de modernización en Tampico nace con el proyecto porfiriano para el desarrollo del ferrocarril y tuvo continuidad en la explotación petrolera. En este contexto, el desarrollo de la ciudad se despliega simultáneamente con una fuerte inversión en el tendido de redes de transporte, de energía y servicios para el desarrollo industrial (Bartorila y Loredo, 2017). El patrimonio arquitectónico heredado por el ferrocarril, la industria petrolera y portuaria en Tampico es indiscutible, al menos para académicos e investigadores. Una extensa cantidad de kilómetros de vías, espacios ferroviarios, edificios de equipamiento e infraestructura son testigos del momento histórico más importante del puerto. Sin embargo, el gran reto hoy es la revalorización social de este patrimonio mediante la reconstrucción de la línea hereditaria que el abandono y el desuso, primero del ferrocarril y luego de las instalaciones petroleras, han roto a lo largo de las últimas décadas. Concientizar a la población local de la relevancia del patrimonio es el primer paso para su revalorización y posible conservación. Ahora bien, el estudio, registro y divulgación de este patrimonio se dificulta por tres razones básicas: las obras van siendo sistemáticamente destruidas, modificadas y reemplazadas, porque al ser un patrimonio moderno no se considera un valor histórico, son apenas construcciones del siglo pasado; las fuentes primarias que detallan las características de las mismas, tales como planos, material bibliográfico, hemerográfico, etc., no se encuentran catalogadas y/o su estado de conservación es pobre, lo que dificulta su lectura o reproducción; y por último, el hecho de que Tampico sea una ciudad periférica dificulta el escenario de gestión de importancia nacional para su preservación tanto a nivel documental como prioritario. Es por ello que la importancia de registrar y gestionar los documentos en disposición de archivos locales, así como analizar, valorar y tratar adecuadamente su conservación física, acceso y preservación digital, contribuirá, de manera sustancial dentro del contexto de la gestión de la información en el siglo XXI, a mejorar el acceso al estudio disciplinar del patrimonio arquitectónico moderno, favorecer los procesos de valorización de su importancia y potenciar la difusión mundial de su patrimonio documental arquitectónico en formato digital.

\section{Método}

El estudio es de tipo descriptivo, integrando un enfoque de método cualitativo sistémico como variación, para definir un caso de gestión de información desde el estudio situacional de la demanda e interés documental, disposición de organización documental y recursos humanos, e infraestructura técnica 
disponible en un archivo para el proceso de preservación digital de una colección documental específica. Se ha hecho uso de las técnicas de entrevista, cuestionario y observación como principal fuente descriptiva de trabajo. Por medio del enfoque sistémico de caso es posible organizar, estructurar e integrar de manera armónica diferentes conocimientos para entender una realidad desde una perspectiva de procesos de gestión de la información. El tema de la preservación digital es casuístico, tanto en la aplicación de metodología o procedimientos de los distintos tipos de procesos digitales (Térmens Graells, 2013).

\section{Metodología}

El trabajo se dividió en tres etapas. En la primera se aplicó un cuestionario de valoración y se realizaron entrevistas a un grupo de 36 arquitectos con perfil de investigador y con posgrado de la Facultad de Arquitectura, Diseño y Urbanismo de la Universidad Autónoma de Tamaulipas, México, durante el periodo de enero a mayo de 2019. En el cuestionario se utilizó la escala Likert ${ }^{1}$ para obtener un nivel de tendencia central de demanda documental en una comunidad designada, como media -tipo de datos o contenido- en frecuencia de consulta, interés, y disponibilidad, y de significado - datos de escala- de prioridades documentales por consulta (Boone y Boone, 2012). Estas tendencias fueron contrastadas por medio de entrevistas personales a participantes del cuestionario para precisar datos y obtener detalles específicos como usuarios.

En la segunda etapa se realizaron entrevistas y visitas de observación al Archivo Histórico de Tampico, entre octubre de 2018 y mayo de 2019, para obtener un registro e inventario a nivel de organización de recursos humanos -organigrama-, y de gestión de fondos documentales específicos sobre el tema de patrimonio documental arquitectónico -tipos y cantidad de colecciones, soportes de almacenamiento físico y su catalogación-, para obtener un levantamiento cuantitativo y de conservación de documentos disponibles como colecciones físicas. En una tercera y última etapa, se realizó un inventario de infraestructura tecnológica disponible en el AHT para evaluar y contrastar las posibilidades de alcance ejecutivo, operativo y técnico de un procedimiento de gestión digital a largo plazo.

El método planteado se enfoca en observar las necesidades de una comunidad designada específica y los aspectos nucleares de la preservación

1 La escala Likert fue desarrollada por Rensis Likert en 1932, como método de evaluaciones sumarias sobre niveles de tendencia de acuerdo y desacuerdo sobre un elemento, ítem o pregunta. 
digital sugeridos por McGovern (2007), los cuales se integran por la infraestructura organizacional, la infraestructura tecnológica y los recursos requeridos para desarrollarla. La Figura 1 sitúa los elementos e hitos utilizados como propuesta de modelo para guiar la gestión y valoración preliminar de colecciones de documentos físicos, digitalizados o de origen digital para su preservación digital.

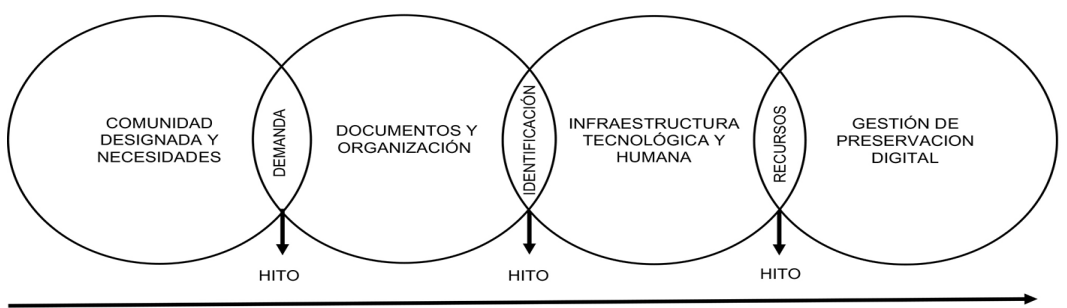

BASE DE ELEMENTOS E HITOS EN PRESERVACIÓN DIGITAL

Figura 1. Modelo de orientación preliminar e hitos para la gestión de preservación digital Fuente: elaboración propia

El modelo de orientación preliminar e bitos para la gestión de preservación digital se integra por cuatro elementos que refieren a personas, objetos y acciones de consideración para el estudio, y por tres hitos o momentos de valoración intermedia, que se enfocan en acciones y objetos de contraste para la valoración. El primer elemento, denominado comunidad designada y sus necesidades, se refiere a un grupo específico de personas interesadas o de usuarios finales con necesidades puntuales de consulta, acceso y disponibilidad de colecciones específicas de una fuente documental de archivo. El segundo elemento lo componen los documentos y su organización, los cuales de manera intrínseca cumplen los protocolos de un sistema de archivo y encuentran composiciones de inventario, orden y gestión documental física o electrónica. El tercer elemento se enfoca en la infraestructura tecnológica y bumana que hace servir a un archivo en su operación e implementación técnica, así como en la definición de roles y actividades en su administración. El cuarto elemento es la gestión de preservación digital, que integra políticas, estrategias y acciones como procesos de buenas prácticas para el tratamiento de un archivo digital y su conservación a largo plazo.

El primer hito del modelo es la demanda, que busca definir un contraste de los contenidos o colecciones de mayor consulta, interés y disposición. El segundo hito es la identificación, que sugiere reconocer y distinguir documentos, personal y roles de los documentos de un archivo en contraste con 
la demanda de la comunidad designada y sus necesidades. El tercer hito es recursos, que define un inventario real de disposición en infraestructura tecnológica y humana ante la gestión de preservación digital.

\section{RESULTADOS Y DiscusióN}

\section{La comunidad designada y necesidades}

La gestión de un programa inicial de curaduría digital, o procedimiento de perfilar, seleccionar y enriquecer un objeto de cara a su gestión de preservación digital, requiere -como punto de partida- de observar las prioridades de elección e interés en algún tipo de contenido o documentos para una comunidad específica, lo que mejora la contextualización comunitaria y una descripción electrónica o folksonomy con la ayuda y participación de la comunidad de usuarios (Brayner, 2019); esto contribuye en la definición de necesidades específicas de contenidos y colecciones, así como en la posibilidad de demanda e identificación social del interés en una comunidad para enfocar las acciones, recursos y alcances en actividades preliminares alrededor de la preservación digital de colecciones específicas.

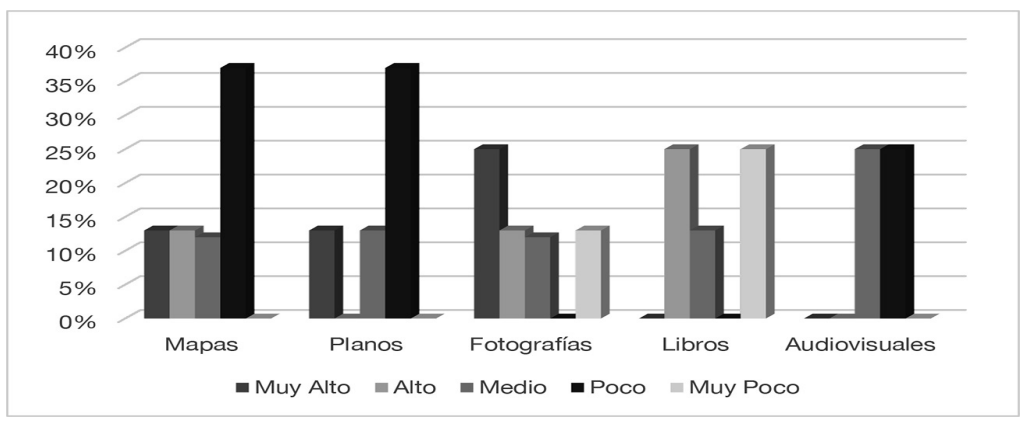

Figura 2. Frecuencia de consulta (FC) de tipos de documentos sobre arquitectura moderna de Tampico en el Archivo Histórico de Tampico Fuente: elaboración propia

En la Figura 2 podemos observar que la frecuencia de consulta muestra un alto índice hacia el nivel Poco en la consulta de mapas, planos y audiovisuales, situándolos entre $37 \%$ y $25 \%$ de los usuarios. Sin embargo, los resultados dejan ver una tendencia polarizada de documentos en la escala de consulta Muy alto, el cual sitúa y beneficia a los tipos de documentos fotográficos, planos y mapas con porcentajes de $13 \%$ y $25 \%$. De acuerdo con 
el contraste de las entrevistas realizadas a los participantes, se observa que la poca consulta de estos documentos está relacionada con la falta de accesibilidad física y espacial a los documentos en el AHT. Cabe señalar que en los resultados se distingue como Alto el nivel de consulta en los mapas y fotografías; lo cual deja ver un escenario de preferencia.

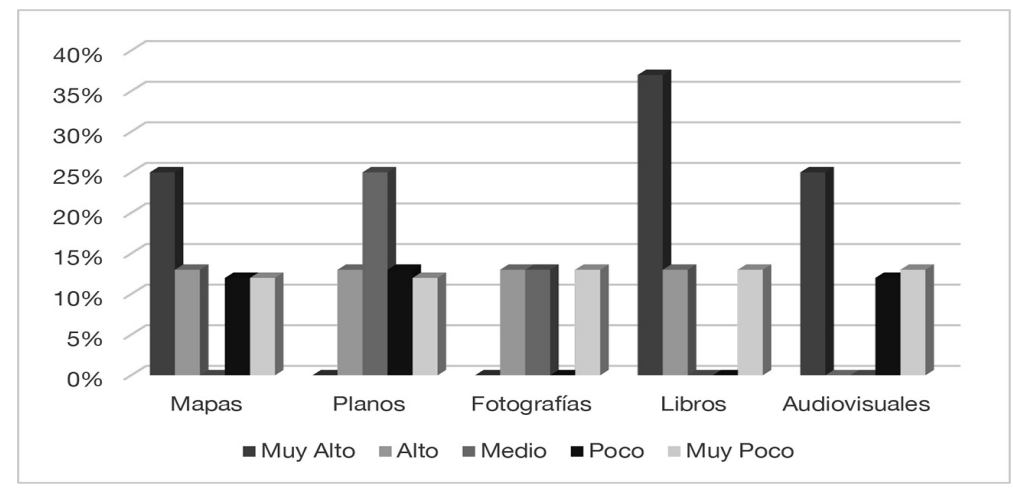

Figura 3. Nivel de interés (IN) en tipos de documentos sobre arquitectura moderna de Tampico en el Archivo Histórico de Tampico

Fuente: elaboración propia

En la Figura 3 se observa una frecuencia de interés Muy alto en los tipos de documentos libros, mapas y audiovisuales, con $37 \%$ y $25 \%$. A nivel Medio de interés destacan los planos y fotografías. En contraste con las entrevistas de participantes, se obtiene que el nivel de interés es un factor de deseo por encontrar información en esos tipos de documentos o soportes, por lo que la tendencia sigue apuntando hacia mapas, planos y fotografías de arquitectura moderna en AHT.

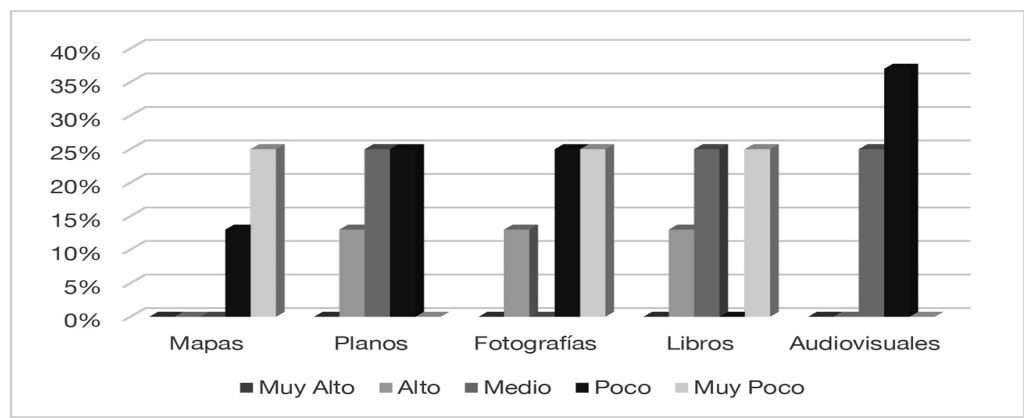

Figura 4. Nivel de disponibilidad (DIS) de tipos de documentos sobre arquitectura moderna de Tampico en el Archivo Histórico de Tampico

Fuente: elaboración propia 
A nivel de valoración y percepción de disponibilidad (Figura 4), los mapas, fotografías y libros puntúan con Muy poco de disponibilidad en $25 \%$ compartido, lo que puede dejar ver un escenario de cruce entre el interés medio y de su consulta ante la disponibilidad con el grado de demanda tipológica ante su acceso; esta tendencia es seguida por los planos y las fotografías con $25 \%$ Poco disponible. En contraste con las entrevistas, se reitera que estos tipos de documentos sobre arquitectura en el AHT mantienen una percepción de no contar con las condiciones de consulta apropiadas y cuyo acceso se ve limitado a estas condiciones de organización.

A nivel de comunidad designada y sus necesidades como usuarios, los resultados del cuestionario respecto de la frecuencia de consulta, el interés y la disponibilidad documental en documentos y colecciones de arquitectura en el Archivo Histórico de Tampico (AHT) nos otorgan un escenario preliminar para priorizar la gestión específica de documentos de interés y discriminar documentos -y colecciones de éstos- en el procedimiento de preparación, organización y estructuración digital. Las definiciones de necesidades y demanda en colecciones concretas de los usuarios son relevantes para los procesos de preservación digital, ya que proporcionan un enfoque de interés y prioridades documentales para iniciar con colecciones precisas; no se puede preservar todo un archivo, pero sí colecciones concretas e importantes para una comunidad designada. Por otro lado, la demanda de colecciones específicas de una comunidad designada abre oportunidades tanto para acciones colaborativas de interés de una comunidad y un archivo de preservación digital, como para establecer actividades de cooperación en la descripción digital o desarrollo de metadatos de los objetos de manera conjunta.

\section{Los documentos y su organización}

Los registros de inventario cuantitativo de documentos sobre la temática de arquitectura existentes y proporcionados por el Archivo Histórico de Tampico se concretan en la Tabla 1.

\begin{tabular}{|l|r|}
\hline \multicolumn{1}{|c|}{ Tipo de documento } & Cantidad disponible en AHT \\
\hline Plano & 209 \\
\hline Fotografía & 30 \\
\hline Mapa (Zonificación) & 22 \\
\hline Libro & 3 \\
\hline Audiovisual & 0 \\
\hline Total & $\mathbf{2 6 4}$ \\
\hline
\end{tabular}

Tabla 1. Inventario de tipos de documentos relativos al tema de arquitectura en Tampico disponibles en el AHT

Fuente: Archivo Histórico de Tampico 
Estas cantidades nos indican una coherencia ocasional, con los resultados de la percepción de demanda de los usuarios o comunidad designada en ese tipo de documentos temáticos. A nivel de organización de documentos sobre la arquitectura en el AHT, hemos encontrado dos niveles: el primero refiere a la organización de documentos y el segundo a la organización del personal del archivo. En este sentido, los planos y fotografías son organizados y almacenados en bolsas de papel o sobres, su inventario está descrito en hojas de cálculo ofimáticas sin esquemas de metadatos visibles, y la clasificación es temática -sin orden cronológico-. Dado que el objetivo de este estudio refiere a establecer orientaciones iniciales para preparar documentos físicos para un proceso de preservación digital, será necesario que el archivo establezca y concrete previamente lineamientos claros sobre la organización, clasificación, catalogación y conservación de documentos físicos, ya que éstos otorgan una sólida identificación documental física y descriptiva sobre las características de los documentos, tales como su calidad, legibilidad, estado de conservación, dimensiones espaciales, pluralidad de formatos, relevancia y demanda del fondo como antesala de su conversión digital.

La guía de archivos personales sugerida por la Biblioteca del Congreso (http://digitalpreservation.gov/personalarchiving/) y traducida como IDOA (identificar, decidir, organizar y almacenar) siguiere acciones prácticas de preparación inicial de documentos digitales de patrimonio personal para su preservación; con esto se abren posibilidades simplificadas de identificación, organización y toma de decisiones aplicables, incluso, a colecciones de documentos físicos que permitan plantear un escenario de conciencia preliminar y cultural ante las actividades futuras de preservación digital.

La organización del personal del AHT como recursos humanos se integra por un equipo de cuatro personas con nombramientos administrativos (Figura 5).

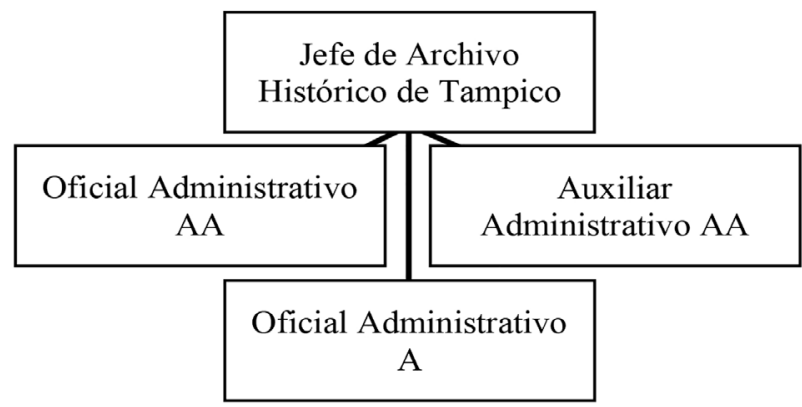

Figura 5. Organigrama y nombramientos de personal del Archivo Histórico de Tampico Fuente: elaboración propia 
La disponibilidad de cuatro personas como recurso humano del AHT puede ser suficiente para afrontar actividades preliminares como la catalogación de colecciones específicas, descripción digital offline de objetos físicos - metadatos- con herramientas de libre acceso como Dublin Core Generator (www.dublincoregenerator.com), así como la investigación y definición de criterios de digitalización para derivados de preservación y difusión. Para ello se deberá elaborar un esquema de roles de trabajo a nivel ejecutivo (dirección de actividades, políticas, gestionar recursos y sostener la actividad), operativo (criterios de digitalización, normalización, descripción y procedimientos) y técnico (configuración de servidores, almacenamiento, instalación de software o contratación de servicios de terceros).

\section{Infraestructura tecnológica}

Los procesos de preservación de objetos de origen digital o digitalizados requieren de herramientas tecnológicas e infraestructuras técnicas a nivel de hardware y software para facilitar que las actividades ejecutivas y operativas converjan en un sistema pleno de preservación digital, que automatice servicios de preservación, difusión y almacenamiento digital. El inventario de infraestructura o equipo tecnológico disponible en el AHT se define en la Tabla 2.

\begin{tabular}{|l|c|l|}
\hline \multicolumn{1}{|c|}{ Tipo de equipo } & Cantidad & \multicolumn{1}{c|}{ Uso principal } \\
\hline Computadoras de escritorio & 3 & Creación de documentos administrativos \\
\hline Multifuncional (impresora y escáner) & 1 & Impresión de documentos administrativos \\
\hline Conexión a internet & 1 & Correo electrónico y difusión de actividades \\
\hline Disco de almacenamiento digital externo & 2 & Respaldo de hemeroteca digitalizada \\
\hline
\end{tabular}

Tabla 2. Tipo de equipo disponible como infraestructura tecnológica en el AHT

Fuente: elaboración propia

De acuerdo con las entrevistas realizadas al personal sobre la infraestructura tecnológica y el equipo disponible, los participantes coinciden en la falta de actualización de su equipo y en la necesidad de formaciones técnicas sobre competencias digitales que les permitan ofrecer nuevos servicios a los usuarios del archivo mediante el aprovechamiento de las prestaciones que brindan las tecnologías de la información. Debemos acentuar que una infraestructura tecnológica limitada local no representa un obstáculo para trabajar en preservación digital, ya que la infraestructura humana puede trabajar en avanzada con esquemas de planificación de políticas, procedimientos y estándares necesarios para orientar la cobertura funcional de la dimensión técnica de preservación digital. 
De acuerdo con autores como Voutssás (2012), la mayoría de los programas de preservación digital requieren de $90 \%$ de trabajo destinado a actividades de planificación, y sólo 10 \% a las implementaciones de soluciones técnicas. Estas orientaciones tienen sentido, ya que las herramientas técnicas de hardware y software en preservación digital han evolucionado tanto en su opción "hágalo usted mismo" con software libre como en su oferta de sistemas con servicios plenos de preservación digital que están listos para usarse de forma simplificada con software propietario, los cuales, de acuerdo a necesidades, limitaciones y alcance de cada organización, ofrecen soluciones de cobertura técnica con posibilidades de contratación externa a modo de legado, licencias y servicios integrales, ofreciendo software todo en uno que integra repositorios de preservación y difusión con contendedores que utilizan inteligencia artificial para el almacenamiento dedicado y controlado de preservación digital en la nube, sin ocupar ni invertir en infraestructura propia para ello.

Acorde con entrevistas y observaciones al personal del AHT, se ha comprobado que dos miembros del personal tienen conocimientos operativos sobre procedimientos de digitalización de documentos de oficina con equipos genéricos -multifuncionales de escala doméstica- de marcas comerciales. La infraestructura técnica para procesos de digitalización de documentos físicos también puede ser externalizada, siempre que se siga un esquema de requerimientos estandarizados con las guías FADGI (Federal Agencies Digital Guidelines Initiative, http://www.digitizationguidelines.gov), Metamorfoze (https://www.metamorfoze.nl/) y la norma ISO 19264-1 (Photography - Archiving systems - Image quality analysis, https://www.iso.org/ standard/64221.html) para cubrir la calidad de digitalización para preservación y difusión a demanda. Con respecto al conocimiento de temas relativos a políticas, estrategias y acciones de preservación digital, el equipo responsable del AHT menciona en su mayoría que desconocen sobre éstos, pero están interesados en ir preparándose para ello.

\section{Conclusiones}

Conscientes de las limitantes a nivel de infraestructura tecnológica, de organización documental y de recursos humanos registrados en el Archivo Histórico de Tampico, hemos constatado y concluido que la aplicación del modelo de orientación preliminar e hitos para la gestión de preservación digital ofrece una alternativa simplificada y asequible para guiar las acciones de observación y estudio que recopilan información específica de contraste y de caso sobre 
demandas documentales tipológicas de documentos, la identificación y el estado de colecciones documentales, así como de los recursos e infraestructura disponibles para su gestión documental física o digital; esta información es de utilidad como orientación preliminar de borradores o bocetos de documentos de políticas y procedimientos de preservación digital necesarios para un caso específico de archivo.

El estudio aplicado a una comunidad designada colabora en mitigar las indecisiones sobre qué tipo de colecciones son prioritarias para un archivo -política de preservación-, ya que de acuerdo con el interés y demanda de una comunidad designada o varias, se puede mejorar un sistema de decisión y evaluación transversal de las demandas de colecciones posibles a preservar digitalmente.

Sobre el énfasis en los documentos y su organización, se ha observado que se puede obtener una orientación minorista de identificación y discriminación funcional sobre los tipos de documentos, clasificación y estado de disponibilidad, lo que da como resultado un proceso de pre-curaduría digital de las colecciones prioritarias elegidas por la comunidad designada como proceso previo a su digitalización, difusión y preservación.

La identificación de la infraestructura humana y tecnológica disponible nos da información sobre un equipo real y sólido para considerar las posibilidades de alcances generales de actividades preliminares de preservación digital situadas en la dimensión ejecutiva (políticas, investigación de estándares y recursos) y operativa (procedimientos, estrategias y acciones necesarias), antes de implementar infraestructuras tecnológicas que cubran la dimensión técnica de la preservación digital.

La visualización preliminar de información sobre elementos e hitos pone en evidencia un escenario realista para priorizar actividades que eviten la procrastinación en preservación digital y permitan la planificación preliminar de políticas y acciones de preservación digital necesarias para orientar la calidad digital del contenido con procedimientos, prestaciones y servicios de preservación, difusión y acceso digital a colecciones específicas en formatos digitales con estándares de preservación desde su origen y creación, antes de implementar herramientas tecnológicas que someten y frenan, en muchas ocasiones, a instituciones con recursos limitados para iniciar procesos de gestión o administración de patrimonio digital, algo que inevitablemente deberán abordar en algún momento de este siglo XXI. 


\section{Consideraciones}

Es importante mencionar que este estudio fue guiado por un modelo teórico y práctico para observar y recoger información previa a un procedimiento formal de preservación digital, por lo que se pueden encontrar limitantes y sesgos -intereses, consultas, demandas, etc.- que nos pueden llevar a obtener planteamientos genéricos en algunos casos; para ello consideramos de vital importancia que el trabajo de campo se desarrolle de manera colaborativa y controlada entre investigadores y el personal del archivo, para evitar observaciones subjetivas que puedan dejar al margen necesidades y soluciones reales. En esta línea, apuntamos que el uso correcto de entrevistas personales y una observación documentada rigurosamente ofrecerán un contexto enriquecido acorde a la realidad, evitando y esclareciendo los límites de su privacidad operativa.

Es indudable que las colecciones documentales alojadas en el AHT referentes al ámbito histórico de la arquitectura moderna de Tampico, como mapas, planos y registros fotográficos, representan y tienen un alto valor para comunidades de estudios académicos relativos a la arquitectura, por lo que su organización de cara a su transformación y preservación digital ayudará a mejorar su accesibilidad y legibilidad y a que funcionen como herramienta con servicios de consulta de información para complementar las necesidades de comunidades designadas, actuales y futuras, con la ayuda de plataformas y recursos digitales bien planificados en la oferta de los sistemas de archivos.

\section{REFERENCIAS}

Arreola Semadeni, Haidy. 2012. "Repositorios de tesis digitales de posgrado: Análisis en trece instituciones mexicanas". Tesis de maestría, UNAM, Biblioteconomía y Estudios de la Información, México.

Boone, Harry y Deborah Boone. 2012. "Analyzing likert data". Journal of Extension $201250(2): 1-5$. https://www.joe.org/joe/2012april/tt2.php

Bartorila, Miguel y Reina Loredo. 2017. "La Industria Petrolera y la Modernidad: Transformaciones Urbanas en Tampico-Madero, Tamaulipas, México". Contexto. Revista de la Facultad de Arquitectura de la Universidad Autónoma de Nuevo León 11 (14): 43-61.

Brayner, Aquiles. 2019. "Digital curatorship: new models of public participation in the description of contents in cultural institutions". Revista Ibero-Americana de Ciência da Informação 12 (1): 53-65. https://doi.org/10.26512/rici.v11.n3.2018.10521

Corrado, Edward. 2019. "Repositories, Trust, and the CoreTrustSeal". Routledge Journals 36 (1): 61-72. https://doi.org/10.1080/07317131.2018.1532055 
Gamboa Ramírez, Ricardo y Rosario Gabriela Páez Flores. 2012. "Petróleo y crecimiento urbano: un pasaje de la historia de Tampico en el siglo XX”, en Historia Económica Regional III, editado por Laura Hernández Montemayor, Mercedes Certucha Llano y Luis Anaya Merchant, 135-157. Cd. Victoria: Fomento Editorial UAT.

Hirtle, Peter. 2008. "The history and current state of digital preservation in the United States", en Metadata and Digital Collections: A Festschrift in Honor of Tom Turner, editado por Elaine Westbrooks y Keith Jenkins, 121-140. Estados Unidos: CIP (CU Library Iniatives in Publishing).

Keefer Riva, Alic y Núria Gallart Marsillas, 2007. La preservación de recursos digitales: El reto para las bibliotecas del siglo XXI. España: Editorial UOC.

Leija, David. 2017. "Preservación digital distribuida y la colaboración interinstitucional: Modelo de preservación digital para documentos con fines de investigación en universidades de México.” Tesis doctoral, Universidad de Barcelona, Facultad de Biblioteconomía y Documentación.

Leija, David y Miguel Márdero. 2019. "Competencias y perspectiva de acciones de preservación digital en Latinoamérica”. Inclusão Social 13 (1): 66-77.

McGovern, Nancy. 2007. "A Digital Decade: Where Have We Been and Where Are We Going in Digital Preservation?” RLG Diginews 11 (1).

Riguzzi, Paolo. 1999. "Mercados, regiones y capitales en los ferrocarriles de propiedad mexicana, 1870-1908”, en Ferrocarriles y obras públicas, editado por Sandra Kuntz Ficker y Priscilla Connolly, 39-52. Ciudad de México: Instituto Mora, El Colegio de Michoacán, El Colegio de México, Instituto de Investigaciones Históricas-UNAM.

Rodríguez, Perla. 2019. "Bibliotecas, Archivos, Museos y Galerias (BAMG) ante la preservación digital de la herencia documental: una aproximación a problemas y soluciones comunes", en Agendas Internacionales de Información y su repercusión en los Estudios de la información, coordinado por Egbert John Sánchez Vanderkast, 179-193. Ciudad de México: IIBI-UNAM.

Térmens Graells, Miquel. 2013. Preservación digital. España: Editorial UOC.

Voutssas, Juan. 2010. "Factores tecnológicos, legales y documentales de la preservación documental digital”. Investigación Bibliotecológica: archivonomía, bibliotecología e información 23 (49): 67-124.

http://doi.org/10.22201/iibi.0187358xp.2009.49.21391

Voutssás, Juan. 2012. "Preservación del patrimonio documental digital en el mundo y en México". Investigación Bibliotecológica: archivonomía, bibliotecología e información 26 (56): 71-113.

http://doi.org/10.22201/iibi.0187358xp.2012.56.33014

Para citar este texto:

Leija Román, David Alonso, Reina Loredo Cansino y Lorena Gertrudis Valle Chavarria. 2020. "Orientaciones preliminares para la preservación digital del patrimonio documental arquitectónico de Tampico”. Investigación Bibliotecológica: archivonomía, bibliotecología e información 34 (85): 13-32.

http://dx.doi.org/10.22201/iibi.24488321xe.2020.85.58182 


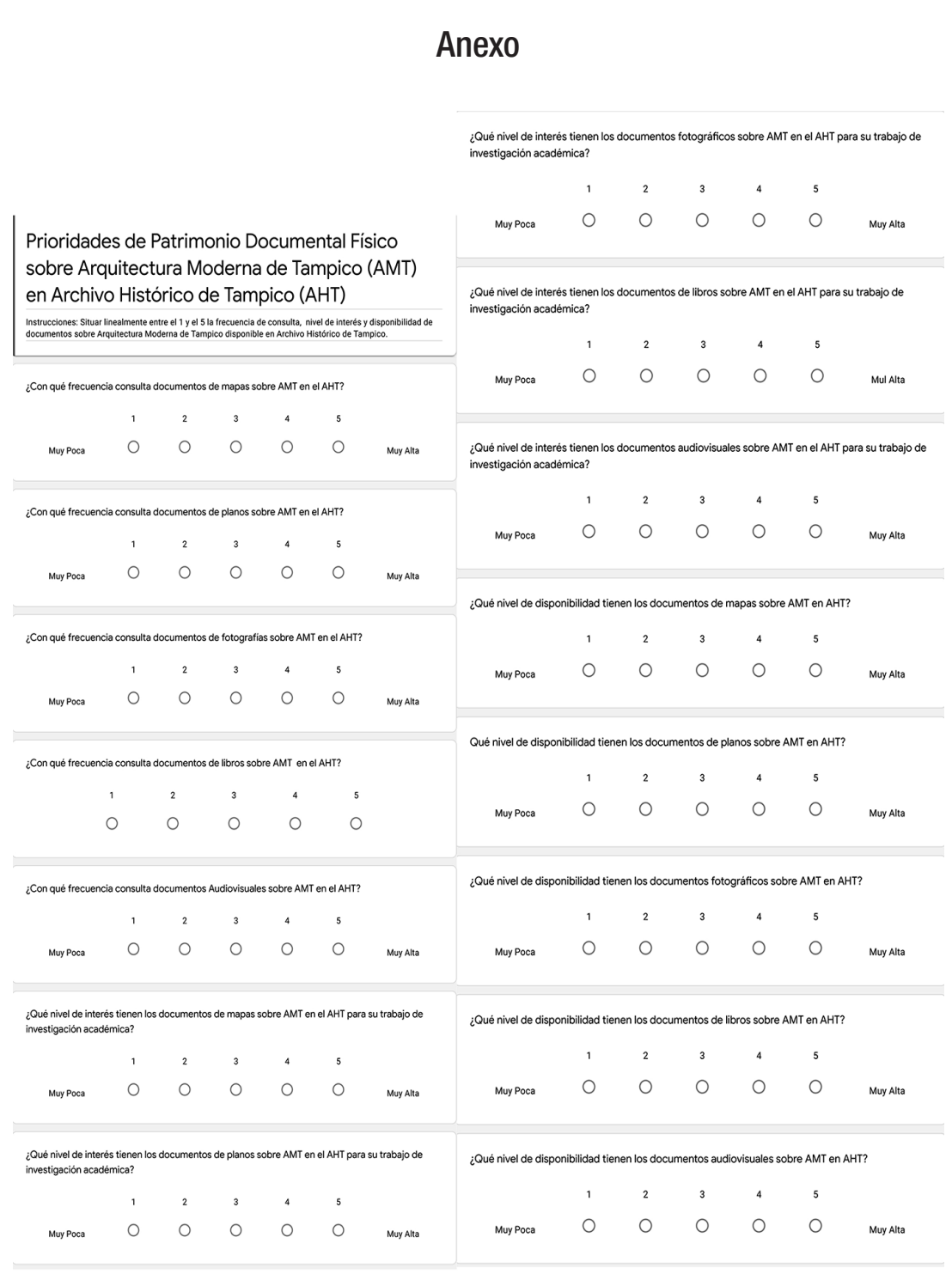

Cuestionario a grupo de expertos / usuarios 


\section{Preguntas para entrevista a participantes de cuestionario}

a) De acuerdo con sus respuestas sobre la frecuencia de consulta de documentos relativos a la arquitectura moderna de Tampico en el archivo histórico de Tampico:

1. ¿Por qué considera usted que su frecuencia de consulta en general de documentos sobre arquitectura en el AHT es: muy baja, baja, media, alta o muy alta (de acuerdo con respuesta)?

2. ¿Qué acciones considera usted podrían ayudar a mejorar su frecuencia de consulta en general de documentos sobre arquitectura en el AHT?

b) De acuerdo con la disponibilidad de documentos relativos a la arquitectura moderna de Tampico:

1. ¿Por qué considera usted que la disponibilidad en general de documentos sobre arquitectura en el AHT es: muy baja, baja, media, alta o muy alta (de acuerdo con respuestas)?

2. ¿Qué acciones considera usted podrían ayudar a mejorar la disponibilidad en general de documentos sobre arquitectura en el AHT?

c) De acuerdo con el interés sobre documentos para su investigación:

1. ¿Por qué considera usted que su interés en general por documentos sobre arquitectura en el AHT es: muy baja, baja, media, alta o muy alta (de acuerdo con respuestas)?

2. ¿Qué acciones considera usted podrían ayudar a mejorar su interés en general de documentos sobre arquitectura en el AHT? 